\title{
Correction to: Automated segmentation of magnetic resonance bone marrow signal: a feasibility study
}

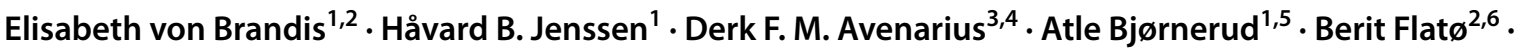 \\ Anders H. Tomterstad ${ }^{1} \cdot$ Vibke Lilleby $^{6} \cdot$ Karen Rosendahl $^{3,4} \cdot$ Tomas Sakinis $^{1,2} \cdot$ Pia K. K. Zadig ${ }^{3,4}$. \\ Lil-Sofie Ording Müller ${ }^{1}$
}

Published online: 22 February 2022

○) Springer-Verlag GmbH Germany, part of Springer Nature 2022

\section{Correction to: Pediatric Radiology (2022) https://doi.org/10.1007/s00247-021-05270-x}

Fig. 1b appeared twice (as Fig. 1a and Fig. 1b) in the original published online version. The correct figure subparts appear below.

Fig. 1 a-c MRI, coronal T2-W Dixon water-only of the knee in a healthy and asymptomatic 14-year-old girl. The perceived intensity level of the periphyseal bone marrow hyperintensity in the distal femur varies considerably with different window $(W)$ and level (C) settings: (a) C192/ W501, (b) C122/W271 and (c) C96/W198
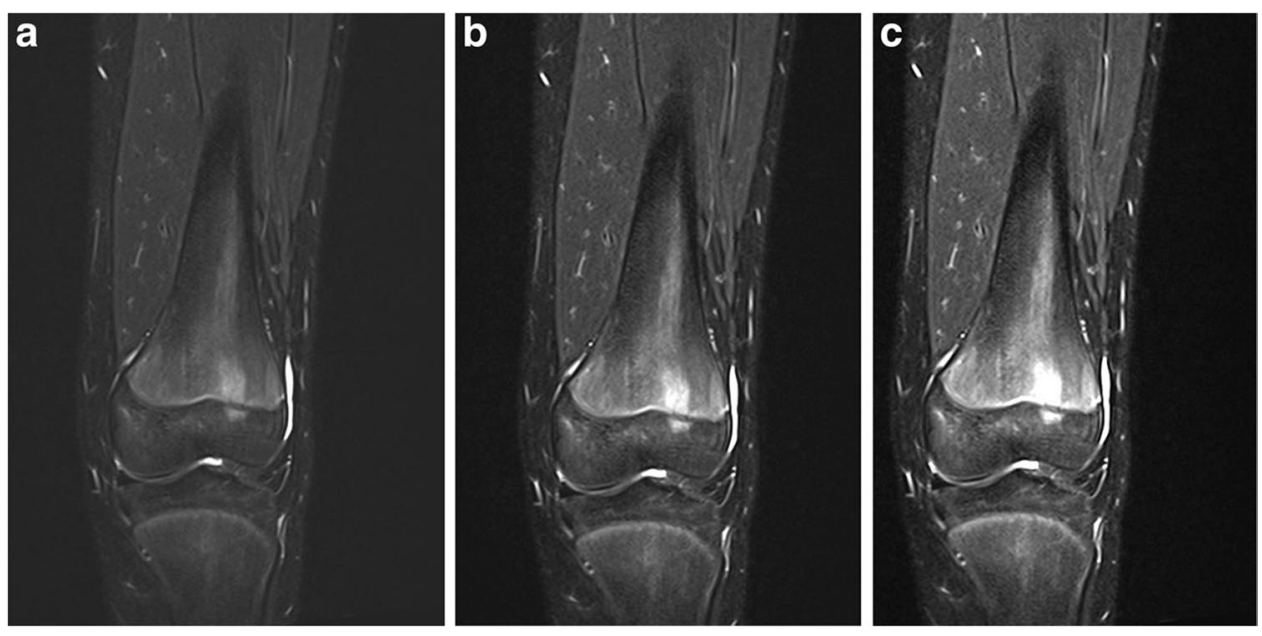

Publisher's note Springer Nature remains neutral with regard to jurisdictional claims in published maps and institutional affiliations.

The original article can be found online at https://doi.org/10.1007/ s00247-021-05270-x

Elisabeth von Brandis

elivon@ous-hf.no

1 Division of Radiology and Nuclear Medicine,

Oslo University Hospital,

Sognsvannsveien 20, 0372 Oslo, Norway

2 Department of Medicine, Institute of Clinical Medicine, University of Oslo, Oslo, Norway
3 Department of Radiology, University Hospital of North-Norway, Tromsø, Norway

4 Department of Health Sciences, University of Troms $\varnothing$, Tromsø, Norway

5 Department of Physics, University of Oslo, Oslo, Norway

6 Department of Rheumatology, Oslo University Hospital, Oslo, Norway 